\title{
Introduction to the Minitrack for Social Impact Organizing and Collaborating
}

\author{
Amber Young \\ University of Arkansas \\ AYoung@walton.uark.edu
}

\author{
Jordana George \\ Texas A\&M University \\ JGeorge@mays.tamu.edu \\ mailto:emailaddress@xxx.xxx
}

\author{
Sirkka Jarvenpaa \\ University of Texas at Austin \\ Sirkka.jarvenpaa@mccombs.utexas.edu
}

In this minitrack we seek to address advances in social impact research in technology-mediated environments. A number of questions arise when considering the social impact of today's information systems and ICTs. How does technology impact collective action? How do indigenous groups promote cultural identity through digital means? How do marginalized people utilize digital technologies to make their voices heard? How do bots and other automation influence politics? How can fake news be mitigated?

The papers selected for this minitrack contribute to the literature on social impact. The first paper starts off delving into fake news. Fake news has become a scourge on digital communications [1], yet we still have much to learn about how and why fake news is currently flourishing. The authors of our first paper, How does Information Spread? A Study of True and Fake News, use the Elaboration Likelihood Model (ELM) to help explain the phenomenon. Asking what makes information believable and what makes people share information, the results of the survey-based study provide some unexpected results. We next move to two papers that explore different perspectives on negative social media behaviors.

Our second paper, Global trolling: The case of "America First," focuses on the phenomenon of global trolling, a negative social media activity undertaken by perpetrators unhampered by international borders [2]. The qualitative study examines video clips from 60 countries that satirically chasten US President Trump's America First campaign rhetoric. The paper's findings on trolling as collective action and cultural statements are novel and interesting.
Our third paper, "They Deserved It": Using the Just World Hypothesis to Understand Victim Blaming, Apathy, and Support on Social Media, takes a look at victims who share misfortunes on social media and receive unexpected backlash or indifference instead of support. Using "Just World" as a theoretical lens [3], the authors suggest that the cognitive dissonance felt by people reading about victims on social media may lead them to justify the action against the victim.

All three of the papers explore novel aspects of social impact organizing and collaborating, whether by formal means or spontaneous informal occurrences. The minitrack chairs hope these works enlighten the topic areas for researchers and stimulate further research into how IS impacts society.

\section{References}

[1] "Digital Wildfires", The Global Risks Report 2018. http://wef.ch/2D45GVa

[2] Cheng, J., M. Bernstein, C. Danescu-NiculescuMizil, and J. Leskovec, "Anyone Can Become a Troll: Causes of Trolling Behavior in Online Discussions", Proceedings of the 2017 ACM Conference on Computer Supported Cooperative Work and Social Computing - CSCW '17, ACM Press (2017), 12171230.

[3] Lerner, M.J., and D.T. Miller, "Just world research and the attribution process: Looking back and ahead", Psychological Bulletin 85(5), 1978, pp. 1030-1051. 